\section{Brave Dreams: An overestimated study, crippled by recruitment failure and misleading conclusions}

\author{
Bernhard H.J. Juurlink, ${ }^{1}$ \\ Pietro M. Bavera, ${ }^{2}$ Salvatore Sclafani, ${ }^{3}$ \\ Ivo Petrov, ${ }^{4}$ Donald B. Reid ${ }^{5}$ \\ ${ }^{1}$ Department of Anatomy \& Cell \\ Biology, College of Medicine, University \\ of Saskatchewan, Saskatoon, SK, \\ Canada; ${ }^{2}$ Vascular Surgeon and \\ Diagnostician Medick-Up Vascular Lab, \\ Milan, Italy and Member of the Italian \\ Society for Angiology and Vascular \\ Medicine (SIAPAV) \& Member of the \\ Italian Society for Vascular Investigation \\ (SIDV) Milan, Italy; ${ }^{3}$ Department of \\ Radiology, SUNY Downstate Medical \\ School, Brooklyn, NY, USA; \\ ${ }^{4}$ Department of Cardiology, Angiology \& \\ Electrophysiology, Cardiovascular \\ Center, Acibadem City Clinic, Sofia, \\ Bulgaria; ${ }^{5}$ The Edinburgh Clinic, \\ Edinburgh, Scotland, UK
}

A recent study, published in JAMA Neurology, examining whether using percutaneous transluminal angioplasty (PTA) to correct chronic cerebrospinal venous insufficiency (CCSVI) in multiple sclerosis (MS) patients concluded: Venous PTA has proven to be a safe but largely ineffective technique; the treatment cannot be recommended in patients with MS. ${ }^{1}$ This is rather a bold statement for a study that was grossly underpowered. Not surprisingly, given the history of reaction to the idea that impairment of venous return might influence the progression of MS, the publication of this study was followed by several editorials that bemoaned the power of social media to influence research on and treatment of disease. $^{2,3}$

The Brave Dreams clinical trial was a multi-centre, randomized, sham-controlled evaluation of the efficacy and safety of venous PTA of extra-cranial and extra-vertebral veins that contributed to CCSVI in patients with MS. ${ }^{1}$ Involved were six centres accredited by the Italian National Health Service. Only physicians trained and accredited in functional outcomes, operation of Echo Colour Doppler (ECD) and catheter venography with and without PTA participated. Patients in the trial were between 18 and 65 years old who had a diagnosis of remitting relapsing (RR) or secondary progressive (SP) MS with Extended Disability Status Scale (EDSS) score between 2 and 5.5, disease duration of
15 years or less, a stable neurology condition for at least 30 days, CCSVI as determined by ECD, not having received MSspecific treatment for at least six months, no prior PTA nor having a history on being on certain medications such as fingolimod.

The primary outcomes measured at 12 months were a functional composite score and MRI-detectable lesions. A new functional composite score was developed based upon commonly-experienced functional impairments such as walking control, balance, manual dexterity, post-void residual urine volume, visual acuity, etc. Patients were evaluated and placed into improved, stable, worsened or mixed categories. MRI analysis grouped patients into categories having new and/or enlarged lesions compared to baseline and those free of lesions. Secondary outcomes included annualized relapse rates, changes in EDSS score and proportion of patients with restored venous flow.

A power analysis was performed that determined that to detect 2.1 fewer lesions in RR MS patients at $90 \%$ power (an $\alpha$ of 0.05 ) would require the enrollment of 423 patients and at an $80 \%$ power would require enrollment of 300 patients. For SP MS patients a $90 \%$ power would require recruiting 222 patients. How many patients were actually enrolled in the clinical trial? Only 115 RR MS patients enrolled in the study, of whom 112 completed the study while only 15 SP MS patients were enrolled. Herein lies the major problem of the study: gross underpowerment. This incomplete study should not have been published, rather additional centres should have been established to ensure adequate patient enrolment.

What the study found was that there were essentially no differences in functional composite score between the PTA and Sham groups of RR MS. However, 73\% of the PTA group had no new gadolinium-enhancing lesions compared to $49 \%$ in the Sham group $(\mathrm{P}=0.08)$. For secondary endpoints the study showed that $23 \%$ of PTA had at least one relapse (annualized rate of 0.32 ) compared to $31 \%$ (annualized rate of 0.39 ) of the Sham group but this was not a significant difference.

With SP MS there were no differences in composite functional score between the two groups; however, $100 \%$ of the PTA group $(\mathrm{n}=10)$ developed no new lesions as opposed to $40 \%$ in the Sham group $(n=5)$.

In summary, there was a trend for fewer new lesions in both the RR and SP MS groups if they had PTA and fewer relapses in the PTA group of RR MS patients. However, there were no differences noted between the two groups for composite functional and EDSS scores. About $41 \%$ of the
Correspondence: Bernhard H.J. Juurlink, Department of Anatomy \& Cell Biology, College of Medicine, University of Saskatchewan, 683 Butterfield Road, Mill Bay, BC, V0R 2P4, Canada.

Tel: 1-250-815-5656.

E-mail: bernhard.juurlink@usask.ca

Contributions: the initial draft was written by B. Juurlink following suggestions of the other authors. This initial draft was then modified according to the further suggestions of each of the authors.

Conflict of interests: the authors have no conflict of interests to disclose

Received for publication: 12 February $2018 .$.

Revision received: 21 March 2018.

Accepted for publication: 21 March 2018.

This work is licensed under a Creative Commons Attribution 4.0 License (by-nc 4.0).

CCopyright B.H.J. Juurlink et al., 2018

Licensee PAGEPress, Italy

Veins and Lymphatics 2018; 7:7340

doi:10.4081/vl.2018.7340

RR improved compared to $49 \%$ of the Sham while $12 \%$ of the RR and $19 \%$ of the Sham worsened with the remaining patients showing a mixed outcome. Curiously, median EDSS scores decreased from a median score of 2.5 to 2.0 in both the PTA and Sham-treated groups.

What would the results have been if the study was properly powered? We point out that in a large study where 366 MS patients who had PTA to correct for CCSVI were followed up for 4 years, PTA resulted in significant clinical improvement, especially in the RR MS patient group. ${ }^{4,5}$ The patients were divided into RR (264), Primary Progressive (PP) and SP groups. All patients underwent a Duplex exam and filled out a Questionnaire that addressed the following symptoms: diplopia, fatigue, headache, upper limb numbness/mobility, lower limb numbness/mobility, altered thermic sensibility, bladder control, balance coordination, quality of sleep, vertigo, mind concentration and working activity. Patients with CCSVI then underwent PTA and were followed up for 4 years. It is important to note that the researcher carrying out the Duplex exams and analyzing the Questionnaire data was completely independent of the vascular surgeons carrying out the PTA. This large study demonstrated that in RR MS patients that venous blood flow improvements were long-lasting when the abnormalities were not so severe. Further, improved venous outflow was 
associated with long-lasting improvements in clinical symptoms with improvements in: more than $90 \%$ with diplopia, fatigue, headache, quality of sleep, vertigo and ability to mentally concentrate; more than $80 \%$ in balance control and upper and lower limb functions; and more than $65 \%$ in bladder control and thermic sensibility. In contrast, although SP and PP MS patients showed some initial clinical improvements following angioplasty, these disappeared within 212 weeks.

A problem with the Brave Dreams study is that only about half of the patients had improved venous blood flow following PTA. Clearly, the reasons underlying this surprising failure to improve blood flow in almost $50 \%$ of the patients treated for CCSVI must be investigated. The authors are encouraged to publish a review of the technical methodology and outcomes of their study so that the techniques can be analyzed and enhancement in technique be considered. It is equally important to delineate which subset of MS patients respond to PTA. We point out that carotid endarterectomy, which now is a well-accepted common stroke prevention technique in a subset of patients, was questioned as recently as $1984 .{ }^{6}$ The challenge in determining efficacy of endarterectomy was to define which subset of patients benefited from the surgery. This has now been clarified. ${ }^{7}$ Those who have performed angioplasty to correct for CCSVI in MS patients have noted that only a subset of patients benefits from treatment. More research is needed to identify the subset of MS patients with CCSVI that can benefit from the treatment.

What also struck us was the lack of composite functional endpoint analysis of the subset of PTA-treated patients (54\%) where blood flow improved compared to the patients where blood flow was not improved? After all, one of the objectives of PTA in treating CCSVI is improvement of venous outflow and cerebrospinal fluid drainage to ultimately enhance cerebrospinal perfusion. And, as noted above, in the Bavera follow-up study clinical improvements were noted only if there were improvements in venous outflow following PTA. ${ }^{4,5}$ Further, why was no attention paid to the fact that $38 \%$ of the sham-treated group had improved blood flow, after all improved blood flow regardless of treatment is desired to improve symptoms of MS. Improved blood flow following shamtreatment is, at first glance, surprising; perhaps valvular and other endoluminal alterations resulting from catheterization itself may improve flow. Moreover, there is some evidence suggesting that PTA may improve autonomic function which may itself improve blood flow. ${ }^{8,9}$ If this be the case then it becomes important to know whether improved venous blood flow, regardless of treatment, improves outcomes. This was not addressed in the paper.

The possibility of technical deficiencies must be considered as a contributor to the poor rate of flow restoration in the Brave Dreams study. Reporting clinical outcomes of a new operative procedure without also reporting the technical parameters of the procedure as was recommended by the International Society for Neurovascular Disease (ISNVD) and by the Society of Interventional Radiology prevents real critique of the procedure. ${ }^{10,11}$ Many aspects of this therapy are dependent on the diagnostic findings, such as use of intravascular ultrasound, degree of stenosis, number of extrinsic compressions, incidence of webs, divisum, septum duplication and webs, transit time, stagnation, reflux, and as well upon technique, such as balloon size versus vessel size, end point of angioplasty, pressure of angioplasty, number of inflations, duration of inflation, residual stenosis, incidence of dissection. Without this information, proceduralists cannot assess the validity of the results, or learn why almost half of the patients failed to have improved flow after angioplasty, nor can they develop improvement in techniques.

One firm conclusion from this randomized, blinded study is that PTA to correct for CCSVI is safe. ${ }^{1}$ This is not a new finding since the safety of PTA to correct for CCSVI had been described previously. ${ }^{12} \mathrm{We}$ also note that if one combines the Remitting-Relapsing and the Secondary Progressive MS patients in the PTA ( $n=73$ and $n=10$, respectively) and Sham $(n=37$ and $n=5$, respectively) and examines for absence of new lesion formation, we find that 56/83 PTA patients and 21/42 Sham patients had no new lesions. A Chi Squared analysis shows that the probability of the PTA treatment having no effect is 0.058 . As noted this Brave Dreams study was greatly underpowered and this statistical analysis suggests that on this basis alone further studies are well-warranted and we urge the investigators to continue to enrol patients into their clinical trial and to, especially, dig deeper into the data. There is an abundance of evidence that co-morbidities have an effect on progression to disability in $\mathrm{MS}^{13}$ and it is, therefore, not unreasonable to hypothesize that problems in venous outflow from the CNS would affect progression to disability.

\section{References}

1. Zamboni P, Tesio L, Galimberti L, et al. Efficacy and safety of extracranial vein angioplasty in Multiple Sclerosis. A randomized clinical trial. JAMA Neurol 2018;75:35-43.

2. Green, AJ, Kamel H, Josephson, A. Combating the spread of ineffective medical procedures. A lesson learned from Multiple Sclerosis. JAMA Neurol 2018; 75:15-7.

3. Zivadinov R, Weinstock-Guttman B. Extracranial angioplasty is ineffective in treating MS. Nature Rev Neurol 2018; 14:129-30.

4. Bavera PM. May symptoms of chronic cerebrospinal venous insufficiency be improved by venous angioplasty? An independent 4-year follow up on 366 cases. Veins and Lymphatics 2015; 4:5400.

5. Bavera PM. Chronic Cerebrovascular Vein Insufficiency (CCSVI): how and when can Jugular Vein PTA Influence the most frequent Symptoms and Disturbs in Multiple Sclerosis. Acta Phleb 2016; 17:27-32.

6. Barnett HJM, Plum F, Walton JN. Carotid endarterectomy - an expression of concern. Stroke 1984;15:941-43.

7. Easton JD. History of endarterectomy then and now. Stroke 2014;45:e101-3.

8. Arata M, Sternberg Z. Transvascular autonomic modulation: A modified balloon angioplasty technique for the treatment of autonomic dysfunction in Multiple Sclerosis patients. J Endovasc Ther 2014; 21:417-28.

9. Sternberg Z, Grewal P, Cen S, et al. Blood pressure normalization post-jugular venous balloon angioplasty. Phlebol 2015; 30:280-7.

10. Simka M, Hubbard D, Siddiqui AH, et al. Catheter venography for the assessment of internal jugular veins and azygous vein: Position statement by expert panel of the International Society for Neurovascular Disease. VASA 2013; 42:168-76.

11. Siskin, GP, Haskal ZJ, McLennan G, et al. Development of a research agenda for evaluation of interventional therapies for chronic cerebrospinal venous insufficiency: Proceedings from a multidisciplinary research consensus panel. J Interv Radiol 2011;22:587-93.

12. Petrov I, Grozdinski L, Kaninski G, et al. Safety profile of endovascular treatment for chronic cerebrospinal venous insufficiency in patients with multiple sclerosis. $\mathrm{J}$ Endovasc Therap 2011; 18:314-23.

13. Zhang T, Tremlett H, Zhu F, et al. Effects of physical comorbidities on disability progression in Multiple Sclerosis. Neurol 2018 [Epub ahead of print]. 